\title{
On the performance of Natural Orbital Functional Approximations in Hubbard Model
}

\author{
I. Mitxelena ${ }^{1,2}$, M. Piris ${ }^{1,2,3}$, M. Rodríguez-Mayorga ${ }^{1,4}$ \\ ${ }^{1}$ Donostia International Physics Center (DIPC), 20018 Donostia, Spain. \\ ${ }^{2}$ Kimika Fakultatea, Euskal Herriko Unibertsitatea (UPV/EHU), 20018 Donostia, Spain. \\ ${ }^{3}$ IKERBASQUE, Basque Foundation for Science, 48013 Bilbao, Spain. \\ ${ }^{4}$ Institut de Química Computacional i Catàlisi, 17003 Girona, Spain.
}

\begin{abstract}
Strongly correlated materials are now under intense development, and Natural Orbital Functional (NOF) methods seem to be able to capture the physics of these systems. We present a benchmark based on the Hubbard model for a class of commonly used NOF approximations (also known as reduced density matrix functional approximations). Our findings highlight the importance of imposing ensemble $N$-representability conditions in order to obtain consistent results in systems with either weak or strong electronic correlation, such as the Hubbard system with a varying two-particle interaction parameter. Based on the accuracy of the results obtained by using PNOF7, which retrieves a large amount of the total strong nondynamic correlation, Hubbard model points out that $N$-representability gives solid foundations for NOFs development.
\end{abstract}

\section{INTRODUCTION}

The theoretical foundations of first-order reduced density-matrix functional theory (1RDMFT) were established long time ago [1-4]. Nevertheless, practical approximations which can compete with wavefunctionbased methods have appeared in the last few years [5-17]. 1RDMFT approximations in which the one-electron reduced density matrix (1RDM) plays the central role are given in terms of natural orbitals (NOs) and corresponding occupation numbers (ONs). Hence the functionals tested in this work will be referred to as Natural Orbital Functional approximations (NOFAs), because the corresponding $1 \mathrm{RDM}$ is used in its diagonal representation.

The theorem on the existence of the functional $[1,2,4]$ of the 1RDM does not provide the means for its construction. Exact properties of the hole function [18, 19] have been used to develop NOFAs mantaining the general form of the Hartree-Fock exchange term. These approximations include Coulomb and exchange type integrals, so they are denoted as $\mathcal{J K}$-only NOFAs. Alternatively, Piris has proposed [11] to employ the known ensemble $N$-representability conditions of the two-electron reduced density matrix (2RDM) [20] and progressively enforce them to generate improved functionals. The latter includes exchange and time-inversion integrals [21], so they must be referred to as $\mathcal{J K} \mathcal{K} \mathcal{L}$-only NOFAs.

Recently the interest related to the Hubbard model has grown in electronic structure theory [22-26]. This system can be viewed as the simplest possible model of correlated fermions, since the compromise between kinetic energy, included via particles hopping, and Coulomb repulsion is included in the Hamiltonian. Thus, the Hubbard model exhibits magnetic ordering, metal-insulator transition, superconductivity, and Tomonaga-Luttinger liquid in $1 \mathrm{D}$, among others, so it allows to study many properties in strongly correlated materials. In other words, this minimal model captures the basic nature of electron correlation, and offers full tunability in order to explore different correlation regimes. As such, the Hubbard model is an ideal candidate for calibration and benchmarking of approximate electronic structure methods.

Strongly correlated systems are challenging for theoretical methods, since the independent electron picture fails to describe them. Variational wavefunction approaches such as Gutzwiller [27, 28] and Baeriswyl [2931] have proven to be able to describe fermionic lattice models, and together with DMRG or Quantum MonteCarlo methods, they are commonly employed to study the fundamental properties of strongly correlated lattice systems. An attempt to use another approach based on RDMFT is done here, in fact, appart from the good performance shown by variational $2 \mathrm{RDM}$ calculations $[32,33]$, NOFAs seem also to be suitable to give an accurate description of such systems $[34,35]$. Therefore, the objective of the present paper is to use the Hubbard model as a stringent validation tool for commonly used NOFAs. In particular, the one-dimensional Hubbard model with and without external potential is studied, focusing on energies, double occupancy, and natural ONs. The latter are an indicator of electron correlation [25], so together with the energy results they show how accurate the Hubbard model is being described. The paper is organized as follows. In Sec. II A we describe the main features of NOF theory and we present the approximations employed in this work. Then we give a brief description of the Hubbard model, and we describe the properties that will be studied throughout the paper. Results obtained by using a set of popular NOFAs are shown in Sec. III, together with the exact results obtained from FCI calculations.

\section{THEORY}

\section{A. Natural Orbital Functional Theory}

The Hamiltonian of a many-electron system is the sum of one- and two-particle operators, hence the energy is determined exactly by the 1 and 2 RDM, denoted here- 
after by $\Gamma$ and $D$, respectively. The exact functional can be used with an approximate 2RDM built from 1RDM to obtain an energy functional $E[\Gamma]$. The major advantage of this formulation with respect to the density functional theory (DFT) is that the kinetic energy does not require the construction of a functional. The unknown functional only needs to incorporate electron correlation. This is however a formidable task since the functional $N$ representability [36] which refers to the conditions that guarantee the one-to-one correspondence between $E[\Psi]$ and $E[\Gamma]$, is a problem related to the $N$-representability of the 2RDM [20].

In NOF theory [37], the spectral decomposition of the $\operatorname{RRDM}\left(\Gamma=\sum_{i} f_{i}\left|\phi_{i}\right\rangle\left\langle\phi_{i}\right|\right)$ is used to approximate $E[\Gamma]$ in terms of the NOs $\left\{\phi_{i}\right\}$ and their ONs $\left\{f_{i}\right\}$, namely,

$$
E=\sum_{i} f_{i} \mathcal{H}_{i i}+\sum_{i j k l} D\left[f_{i}, f_{j}, f_{k}, f_{l}\right]<k l|i j\rangle
$$

Here, $\mathcal{H}_{i i}$ is the one-particle part of the Hamiltonian involving the kinetic energy and the external potential operators, whereas $\langle k l \mid i j\rangle$ are the matrix elements of the two-particle interaction. $D\left[f_{i}, f_{j}, f_{k}, f_{l}\right]$ represents the reconstructed $2 \mathrm{RDM}$ from the ONs. We neglect any explicit dependence of $D$ on the NOs themselves because the energy functional (1) has already a strong dependence on the NOs via the one- and two-particle integrals.

It is noteworthy that the resulting approximate functional $E\left[\left\{f_{i}, \phi_{i}\right\}\right]$ can solely be implicitly dependent on $\Gamma[3]$ since the Gilbert's theorem [1] on the existence of the explicit functional $E[\Gamma]$ is valid only for the exact ground-state energy. In this vein, NOs are the orbitals that diagonalize the one-matrix corresponding to an approximate energy that still depends on the 2RDM. A detailed account of the state of the art of the NOF theory can be found elsewhere [38, 39].

We consider $S_{z}$ eigenstates, so only RDM blocks that conserve the number of each spin type are non-vanishing. Specifically, the 1RDM has two nonzero blocks $\Gamma^{\alpha \alpha}$ and $\Gamma^{\beta \beta}$, whereas the $2 \mathrm{RDM}$ has three independent nonzero blocks: $D^{\alpha \alpha}, D^{\alpha \beta}$, and $D^{\beta \beta}$. The parallel-spin components of the $2 \mathrm{RDM}$ must be antisymmetric, but $D^{\alpha \beta}$ presents no special symmetry [37].

We address only singlet states in this work, so the parallel spin blocks of the RDMs are equal. A spin-restricted formalism is adopted, accordingly the NOs have identical spatial parts and equal ONs $f_{i}=f_{i}^{\alpha}=f_{i}^{\beta}$, with $0 \leq f_{i} \leq 1$ and $2 \sum_{i} f_{i}=N$. The latter represents the necessary and sufficient conditions for ensemble $N$ representability of the 1 RDM [40].

The Hartree-Fock (HF) like approximation for the $2 \mathrm{RDM}$ is defined as

$$
\begin{gathered}
D_{i j, k l}^{\sigma \sigma}=\frac{f_{i} f_{j}}{2}\left(\delta_{i k} \delta_{j l}-\delta_{i l} \delta_{j k}\right) \\
D_{i j, k l}^{\alpha \beta}=\frac{f_{i} f_{j}}{2} \delta_{i k} \delta_{j l}
\end{gathered}
$$

Some approximations to the $2 \mathrm{RDM}$ are produced by replacing only the $\sigma \sigma$-elements of the $\mathrm{HF}$ like approxi- mation, namely,

$$
D_{i j, k l}^{\sigma \sigma}=\frac{f_{i} f_{j}}{2} \delta_{i k} \delta_{j l}-\frac{\mathcal{F}\left(f_{i}, f_{j}\right)}{2} \delta_{i l} \delta_{j k}
$$

Table I: $\mathcal{F}\left(f_{i}, f_{j}\right)$ functions used for $2 \mathrm{RDM}$ reconstruction in Eq. (3)

\begin{tabular}{lcc}
\hline Approximation & $\mathcal{F}\left(f_{i}, f_{j}\right)$ & \\
\hline MBB & $\left(f_{i} f_{j}\right)^{1 / 2}$ & {$[5,6]$} \\
Power & $\left(f_{i} f_{j}\right)^{0.53}$ & {$[13]$} \\
CA & $f_{i} f_{j}+\left[f_{i}\left(1-f_{i}\right) f_{j}\left(1-f_{j}\right)\right]^{1 / 2}$ & {$[8]$} \\
CGA & $\frac{1}{2}\left\{f_{i} f_{j}+\left[f_{i}\left(2-f_{i}\right) f_{j}\left(2-f_{j}\right)\right]^{1 / 2}\right\}$ & {$[9]$} \\
\hline
\end{tabular}

The $\mathcal{F}\left(f_{i}, f_{j}\right)$ functions are collected in Table I. These approximations lead to $\mathcal{J K}$-only NOFAs since the electronic energy involves only the Coulomb $\left(\mathcal{J}_{i j}=\langle i j \mid i j\rangle\right)$ and exchange integrals $\left(\mathcal{K}_{i j}=\langle i j \mid j i\rangle\right)$. Note that Eq. (3) violates the antisymmetric requirement unless $\mathcal{F}=$ $f_{i} f_{j}$, consequently none of these functionals affords a $N$ representable 2RDM. Extensive $N$-representability violations for these NOFAs have been reported [41, 42].

The use of the (2,2)-positivity $N$-representability conditions, also known as P, Q, and G conditions, for generating a $N$-representable $2 \mathrm{RDM}$ was proposed by Piris [11]. This particular reconstruction is based on the introduction of two auxiliary matrices $\triangle$ and $\Pi$ expressed in terms of the ONs to reconstruct the cumulant part of 2RDM [43], namely,

$$
\begin{aligned}
& D_{i j, k l}^{\sigma \sigma}=\frac{f_{i} f_{j}-\triangle_{i j}}{2}\left(\delta_{i k} \delta_{j l}-\delta_{i l} \delta_{j k}\right) \\
& D_{i j, k l}^{\alpha \beta}=\frac{f_{i} f_{j}-\triangle_{i j}}{2} \delta_{i k} \delta_{j l}+\frac{\Pi_{i k}}{2} \delta_{i j} \delta_{k l}
\end{aligned}
$$

Both auxiliary matrices are constrained to certain bounds due to the enforced positivity conditions, symmetric properties and sum rules satisfied by the 2RDM. The conservation of the total spin allows to derive the diagonal elements $\Delta_{i i}=f_{i}^{2}$ and $\Pi_{i i}=f_{i}$ [44]. Appropriate approximations for off-diagonal elements lead to different implementations of the NOF known in the literature as PNOFi $(i=1-7)$. The performance of these functionals is comparable to those of best wavefunction-based methods and has been recently reviewed in Ref. [45]. In the present study, the latest functional of this series is used.

PNOF7 [17] corresponds to an interacting electron-pair model. Let us divide the spatial orbital space $\Omega$ into $N / 2$ mutually disjoint subspaces $\Omega_{g}$, so each orbital belongs only to one subspace. Consider each subspace contains one orbital $g$ below the Fermi level $(N / 2)$, and $N_{g}$ orbitals above it, which is reflected in additional sum rules for the ONs:

$$
\sum_{i \in \Omega_{g}} f_{i}=1 ; \quad g=1,2, \ldots, N / 2
$$

Taking into account the spin, each subspace contains solely an electron pair, hence the normalization condition 
for the $1 \mathrm{RDM}$ is automatically fulfilled. It is important to note that orbitals satisfying the pairing conditions (5) are not required to remain fixed throughout the orbital optimization process [46]. Given the functional form of the auxiliary matrices $\triangle$ and $\Pi[17]$, the energy (1) of the PNOF7 can be conveniently written as

$$
\begin{gathered}
E=\sum_{g=1}^{N / 2} E_{g}+\sum_{p \neq q}^{N / 2} E_{p q} \\
E_{g}=\sum_{i \in \Omega_{g}} f_{i}\left(2 \mathcal{H}_{i i}+\mathcal{J}_{i i}\right)+\sum_{i, j \in \Omega_{g}, i \neq j} \Pi_{i j}^{i n t r a} \mathcal{L}_{i j} \\
E_{p q}=\sum_{i \in \Omega_{p}} \sum_{j \in \Omega_{q}}\left[f_{i} f_{j}\left(2 \mathcal{J}_{i j}-\mathcal{K}_{i j}\right)+\Pi_{i j}^{i n t e r} \mathcal{L}_{i j}\right]
\end{gathered}
$$

where

$$
\begin{gathered}
\Pi_{i j}^{\text {intra }}= \begin{cases}-\sqrt{f_{i} f_{j}}, & i \text { or } j \leq N / 2 \\
+\sqrt{f_{i} f_{j}}, & i, j>N / 2\end{cases} \\
\Pi_{i j}^{\text {inter }}= \begin{cases}-\sqrt{f_{i}\left(1-f_{i}\right) f_{j}\left(1-f_{j}\right)}, & i \text { or } j \leq N / 2 \\
+\sqrt{f_{i}\left(1-f_{i}\right) f_{j}\left(1-f_{j}\right)}, & i, j>N / 2\end{cases}
\end{gathered}
$$

From Eq. (6), we can observe that the energy for singlet states contains not only $\mathcal{J}$ and $\mathcal{K}$ integrals, but also the exchange and time-inversion integral $\mathcal{L}_{i j}=\langle i i \mid j j\rangle$ [21], so PNOF7 is a $\mathcal{J K} \mathcal{L}$-only approximation. The first term of the Eq.(6) is the sum of the pair energies described accurately by the two-electron functional $E_{g}$. In the second term, $E_{p q}$ correlates the motion of the electrons in different pairs with parallel and opposite spins. It is clear that the main weaknesses of the approach (6) is the absence of the inter-pair dynamic electron correlation since $\Pi_{i j}^{\text {inter }}$ has significant values only when the ONs differ substantially from 1 and 0 . Consequently, PNOF7 is expected to be able to recover the complete intra-pair, but only the nondynamic inter-pair correlation.

On the other hand, when the $\Pi_{i j}^{i n t e r}$ elements are neglected, Eq. (6) yields the independent pair model PNOF5 [14, 15]. Interestingly, an antisymmetrized product of strongly orthogonal geminals wavefunction with the expansion coefficients explicitly expressed by the ONs also leads to PNOF5 [15], therefore it is strictly $\mathrm{N}$ representable. Indeed, 1RDM functional theory can also be connected to the geminal functional theory [47, 48].

Minimization of the energy functional Eq. (1) is performed under the orthonormality requirement for the NOs, and the particle number fixed to $N$, by using the Lagrange multipliers $\lambda$ and $\mu$ respectively. Occupancies are expressed by the free variable $\gamma$ with $f_{i}=\cos ^{2} \gamma_{i}$ to assure 1RDM ensemble $N$-representability conditions [40]. Thus, we can define an auxiliary functional

$$
\begin{gathered}
\mathcal{L}\left[N,\left\{\gamma_{i}\right\}, \phi_{i}(\boldsymbol{x})\right]=E\left[\left\{\gamma_{i}\right\}, \phi_{i}(\boldsymbol{x})\right] \\
-\mu\left(\sum_{i} \cos ^{2} \gamma_{i}-N\right)-\sum_{i k} \lambda_{i k}\left(\left\langle\phi_{k} \mid \phi_{i}\right\rangle-\delta_{k i}\right)
\end{gathered}
$$

An iterative diagonalization procedure [46] has been employed to make $\mathcal{L}$ stationary with respect to variations in the ONs and the NOs separately, which is based on the hermiticity of the matrix of the Lagrange multipliers $\lambda$ at the extremum. Basically the $1 \mathrm{RDM}$ and $\lambda-\lambda^{\dagger}$, where $\dagger$ denotes the conjugate transpose, can be brought simultaneously to a diagonal form at the solution, hence the set of NOs is given by those orbitals that make $\lambda$ Hermitian. Occupation optimization is done by Sequential Quadratic Programming (SQP) for all $\mathcal{J K}$-only NOFAs. Since both PNOF5 [14] and PNOF7 [17] are electronpairing approaches the particle number is automatically fixed to $N$, so a Conjugate Gradient (CG) algorithm is used to solve the unconstrained occupation optimization in the case of these NOFAs, which yields substantial savings of computational time.

\section{B. Hubbard model}

The Hubbard Hamiltonian is possibly the simplest prototype for modeling strongly correlated systems. This model has been largely used to benchmark electronic structure methods [22-26], but also to describe the electronic properties of many materials, e.g. metal-insulator transitions, charge- and spin-density waves in superlattices, etc. In one dimension and standard notation the Hubbard model Hamiltonian reads

$$
\begin{gathered}
H=-t \sum_{\langle\mu, v\rangle, \sigma}\left(c_{\mu, \sigma}^{\dagger} c_{v, \sigma}+c_{v, \sigma}^{\dagger} c_{\mu, \sigma}\right) \\
+U \sum_{\mu} n_{\mu, \alpha} n_{\mu, \beta}+\sum_{\mu, \sigma} n_{\mu, \sigma} v_{\mu, \sigma}
\end{gathered}
$$

where greek indexes $\mu$ and $v$ denote sites, $\langle\mu, v\rangle$ indicates only near-neighbors hopping, $t>0$ is the hopping parameter, $\sigma=\alpha, \beta, n_{\mu, \sigma}=c_{\mu, \sigma}^{\dagger} c_{\mu, \sigma}$ where $c_{\mu, \sigma}^{\dagger}\left(c_{\mu, \sigma}\right)$ corresponds to fermionic creation(annihilation) operator, $v_{\mu, \sigma}$ is the on-site energy and $U$ is the site interaction parameter. Hereafter, we will refer to as homogeneous Hubbard whenever $v_{\mu, \sigma}=0 ; \forall\{\mu, \sigma\}$, whereas inhomogeneous Hubbard is used whenever non-zero external potential is present. Thus, for the Hubbard dimer it will be set $v_{S_{A}, \sigma}=-v_{S_{B}, \sigma}$ in Sec. III C, so that it gives rise to a nonzero potential $\Delta v$. An even more interesting external potential will be used to study inhomogeneous systems for the 10 sites model, namely the one-dimensional Aubry-André model [49] including electron-electron repulsion

$$
\begin{gathered}
H=-t \sum_{\langle\mu, v\rangle, \sigma}\left(c_{\mu, \sigma}^{\dagger} c_{v, \sigma}+c_{v, \sigma}^{\dagger} c_{\mu, \sigma}\right) \\
+U \sum_{\mu} n_{\mu, \alpha} n_{\mu, \beta}+\sum_{\mu, \sigma} n_{\mu, \sigma} V \cos (2 \pi \alpha \mu+\delta),
\end{gathered}
$$

where $V$ is the modulation amplitude of the on-site potential, $\alpha$ determines the periodicity, and $\delta$ fixes the modulation phase. This model, which is intimately related with the Harper model, has been used to explore topological properties in 1D systems, among others.

The electron-electron repulsion is extremely local in the Hubbard model, and can be tunned by the parameter 
$U$. Although additional complexities can be included by setting variable parameters $U_{\mu}$ and $t_{\mu}$ [22], let us fix $t$ and vary only the particle-particle interaction $U$ in order to cover different correlation regimes, thus $U / t$ will be used as a dimensionless measure for the relative contribution of both terms. In the limit $U / t \rightarrow 0$ the system can be described by mean-field theories, hence the Hartree-Fock approximation recovers the exact wavefunction for the symmetric Hubbard dimer if $U=0$, however, as long as $U / t$ gets larger the system is nevermore weak correlated and methods including electron correlation are needed to give an accurate description of the model.

A general solution for the Hubbard model requires a numerical treatment. We will restrict to half-filling cases throughout this paper, so there is in average one particle per site in the model, such that corresponding distribution among the sites depends on the correlation regime and the number of sites of the Hubbard model. Overall, if the hopping parameter is larger than the on-site interaction the electrons tend to occupy doubly the sites, while at the $U / t \gg 1$ limit, also known as the strong correlation limit, electrons try to keep away one from each other by half-filling the sites, which corresponds to the Mott-Hubbard regime [13, 24]. This transition is quantified by the natural orbital occupancies of the system, $f_{i}$, which actually are an indicator of correlation [25]. Our analysis will focus on the latter, but also on $E / t$ values and on the double occupancy of the sites $d E / d U$, which is defined as

$$
\frac{d E}{d U}=\sum_{\mu}\left\langle\Psi\left|n_{\mu, \alpha} n_{\mu, \beta}\right| \Psi\right\rangle
$$

\section{RESULTS AND DISCUSSIONS}

The Hubbard model is here exploited in order to clarify the performance of commonly used NOFAs in many correlation regimes (specially in the strong correlation regime). The present paper focuses on ground state $E / t$ values, double occupancy (Eq. 11), and natural occupancies in comparison with the exact result; which are obtained from FCI calculations for a range of $U / t$ values in order to cover all correlation regimes in each case. To this purpose we have used a modified version of the code developed by Knowles and Handy [50, 51]. 1RDM and 2RDM have been calculated from the FCI expansion coefficients using a homemade code DMN developed by E. Matito and F. Feixas [52]. The results here presented for all NOFAs have been computed using DoNOF code developed by M. Piris and co-workers. Note that double occupancy of sites has been numerically evaluated by using the five-point formula

$\frac{d E}{d U} \approx \frac{E(U-2 h)-8 E(U-h)+8 E(U+h)-E(U+2 h)}{12 h}$

where the step size is set to $h=0.001$ (the error within this approximation is of the order of $h^{4}$ ).

\section{A. Exact results for homogeneous Hubbard model}

In this section we discuss several properties of the homogeneous 2 sites, 4 sites square, and 6 sites hexagone Hubbard systems, which have been previously employed to study many electronic structure methods [22-26, 53, 54]. Energy values for the homogeneous 10 sites, 12 sites, and 14 sites are also included. Exact FCI $E / t$ values for a range of $U / t$ values corresponding to these systems are shown in Fig. 1.

There is a similar trend for the three curves corresponding to the graph in the left, all of them show negative $E / t$ values at the zero correlation point $(U=0)$ and converge asymptotically to $E / t=0$ at the strongcorrelation limit $(U / t \rightarrow \infty)$. As expected the absolute energies are larger as the number of sites is increased. Interestingly, relative differencies are smaller in the case of 10,12 , and 14 sites systems, and the asymptotic limit is located at larger $U / t$ values when more sites are added to the model.

In order to get a more reliable indicative of the energy, the derivative with respect to parameter $U / t$ is also studied for the 2, 4, and 6 sites systems (Fig. 2), since physical interpretations can be obtained due to their conceptual simplicity. In fact, this is a measure of double occupancy of the sites according to Eq. (11). According to Fig. 2 the double occupancy is maximum in the weak correlation region, since the exact ground-state wave function is recovered by an independent particle model when there are no two-particle interactions. The population of the sites spreads out as the correlation increases, so for large $U$ values the double occupancy tends to zero due to particle-particle repulsion. Neither the energy nor $d E / d U$ show significant differences when the number of sites varies in the Hubbard model, but the study of the ONs displays another situation.

The half-filled 2 sites Hubbard model is the simplest case that we have studied. It is known that when there is no correlation $(U=0)$ the exact wave function for the ground-state may be written as a single Slater determinant $|\Psi\rangle=\left|\sigma_{g}^{2}\right\rangle$ built using a $\sigma_{g}$ orbital; labeled attending to $D_{\infty h}$ symmetry of the system. The $\sigma_{g}$ orbital is defined as

$$
\sigma_{g}=\frac{1}{\sqrt{2}}\left(S_{A}+S_{B}\right)
$$

and is also known as the bonding orbital in quantum chemistry. Note that sites are labeled by $S_{X}$ where $X$ is replaced by a letter in alphabetic order.

Nevertheless, when correlation increases the single Slater determinant picture does not longer hold and the wave function may be written as $|\Psi\rangle=C_{g}\left|\sigma_{g}^{2}\right\rangle+C_{u}\left|\sigma_{u}^{2}\right\rangle$ where the expansion coefficients $C_{g}$ and $C_{u}$ are determined variationally, and the second Slater determinant 

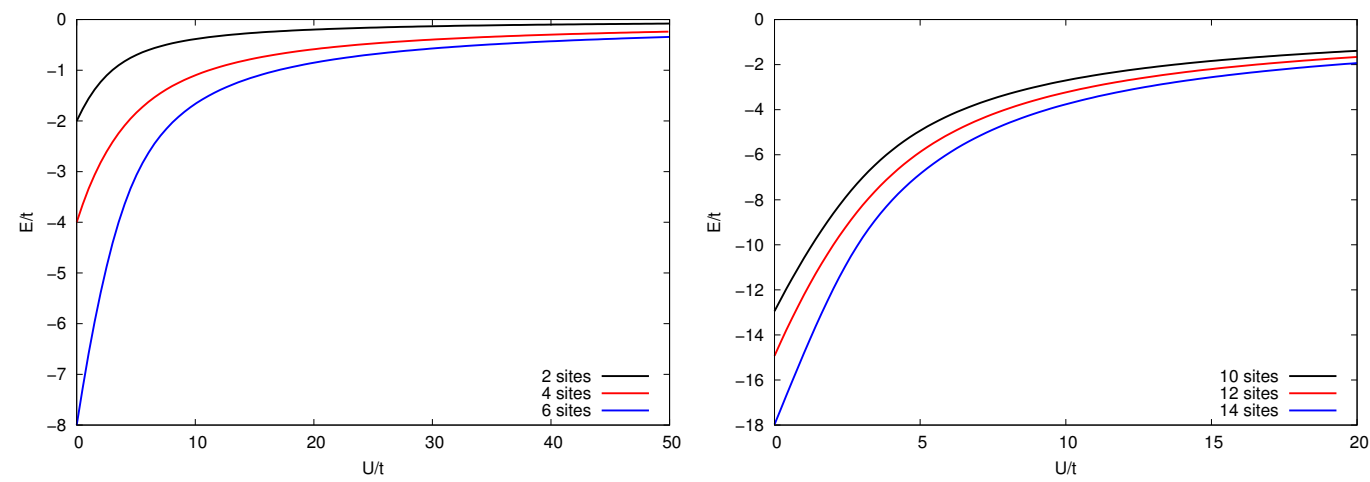

Figure 1: Exact FCI $E / t$ values for many $U / t$ values for half-filled homogeneous $2,4,6,10,12$, and 14 sites Hubbard systems.

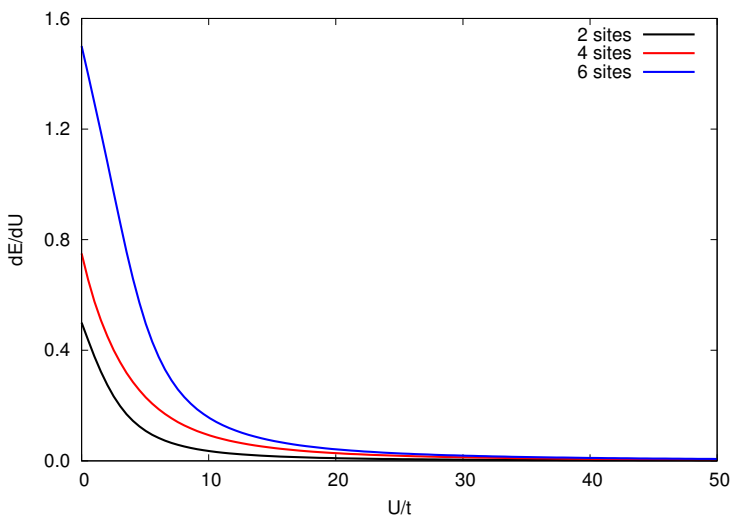

Figure 2: Derivative of the energy with respect to parameter $U / t$ for the homogeneous 2 sites, 4 sites square, and 6 sites hexagone Hubbard models.

is built using the $\sigma_{u}$ orbital

$$
\sigma_{u}=\frac{1}{\sqrt{2}}\left(S_{A}-S_{B}\right)
$$

This orbital is also known as the antibonding orbital in quantum chemistry. The $\sigma_{g}$ and $\sigma_{u}$ orbitals form a basis which is adapted to the symmetry of $D_{\infty h}$ point group. These orbitals are also the NOs for the system since the $1 \mathrm{RDM}$ obtained from the wavefunction is already diagonal in this basis for the homogeneous 2 sites Hubbard model. Corresponding ONs are shown in the top of Fig. 3 . Note that coefficients $C_{g}$ and $C_{u}$ are equalized in the strong-correlation limit $(U / t \rightarrow \infty)$, so the occupancies of the NOs become equal to one (spins summed). In other words, the $1 \mathrm{RDM}$ is diagonal and equal to the identity matrix (this limit resembles the $\mathrm{H}_{2}$ molecule in the dissociation limit for a minimal basis).

For the 4 sites square Hubbard model the orbitals adapted to the $D_{4 h}$ symmetry also correspond to the NOs, these are the non-degenerate

$$
a_{1 g}=\frac{1}{2}\left(S_{A}+S_{B}+S_{C}+S_{D}\right)
$$

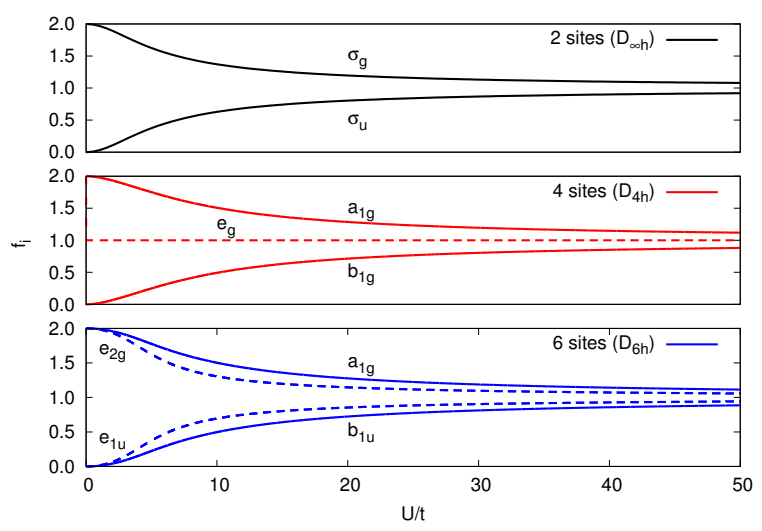

Figure 3: Natural orbital occupancies (spins summed) in terms of $U / t$ values for the homogeneous 2 sites, 4 sites square, and 6 sites hexagone Hubbard models. Solid lines are used for non-degenerate results, whereas dashed lines correspond to degenerate results.

the 2-fold degenerate $e_{g}$ orbitals

$$
\begin{aligned}
& e_{g}(1)=\frac{1}{2}\left(S_{A}-S_{B}-S_{C}+S_{D}\right) \\
& e_{g}(2)=\frac{1}{2}\left(S_{A}+S_{B}-S_{C}-S_{D}\right)
\end{aligned}
$$

and the non-degenerate

$$
b_{1 g}=\frac{1}{2}\left(S_{A}-S_{B}+S_{C}-S_{D}\right)
$$

According to the natural occupancies shown in the middle of Fig. 3, both $e_{g}$ orbitals are half-occupied independently of the interaction strength $U$, whereas orbitals $a_{1 g}$ and $b_{1 g}$ play the role of the $\sigma_{g}$ and $\sigma_{u}$ orbitals in the 2 sites model. Regarding the orbitals adapted to the symmetry of the system in the case of the 6 sites hexagone Hubbard model, which also correspond to the exact NOs, these orbitals read as

$$
a_{1 g}=\frac{1}{\sqrt{6}}\left(S_{A}+S_{B}+S_{C}+S_{D}+S_{E}+S_{F}\right)
$$


the 2 -fold degenerate $e_{2 g}$ orbitals

$$
\begin{gathered}
e_{2 g}(1)=\frac{1}{\sqrt{12}}\left(S_{A}+2 S_{B}+S_{C}-S_{D}-2 S_{E}-S_{F}\right) \\
e_{2 g}(2)=\frac{1}{\sqrt{4}}\left(S_{A}-S_{C}-S_{D}+S_{F}\right)
\end{gathered}
$$

the 2 -fold degenerate $e_{1 u}$ orbitals

$$
\begin{gathered}
e_{1 u}(1)=\frac{1}{\sqrt{4}}\left(S_{A}-S_{C}+S_{D}-S_{F}\right) \\
e_{1 u}(2)=\frac{1}{\sqrt{12}}\left(S_{A}-2 S_{B}+S_{C}+S_{D}-2 S_{E}+S_{F}\right)
\end{gathered}
$$

and the non-degenerate

$$
b_{1 u}=\frac{1}{\sqrt{6}}\left(S_{A}-S_{B}+S_{C}-S_{D}+S_{E}-S_{F}\right)
$$

The plot in the bottom of Fig. 3 shows the exact NO occupancies. The behavior is similar to the one obtained for the 2 sites model; at the weak correlation limit the double occupancy of NOs is maximum, while all orbitals become half-occupied in the limit $U / t \rightarrow \infty$.

\section{B. NOF results for homogeneous Hubbard model}

In this section, the Hubbard model is used as a stringent validation tool for commonly used NOFAs, which have been described in section II A. Note that a spinrestricted formalism has been employed for all calculations in order to hold $\left\langle S^{2}\right\rangle=0$ and $\left\langle S_{z}\right\rangle=0$. Here, we will use the adapted to symmetry basis set described in the previous section for each model, which is conceptually similar to a molecular-like basis set. Interestingly, the latter correspond to the NOs for the homogeneous 2 sites and 4 sites square systems, independently of the interaction strength $U / t$ and the NOFA.

Fig. 4 shows the differences obtained for $E / t$ values with respect to FCI $\left(\Delta[E / t]=E^{N O F A} / t-E^{F C I} / t\right)$ for a wide range of $U / t$ values. As we observe from the left-top of Fig. 4 only MBB, PNOF5 and PNOF7 functionals reproduce the exact FCI energy for the Hubbard dimer (the result for MBB has been previously obtained [26]). Apart from MBB, PNOF5 and PNOF7; CA and CGA functionals show a good asymptotic behavior in the $U / t \rightarrow \infty$ limit. Conversely, neither restricted HartreeFock (RHF) nor the Power functional recover the exact energy or attain a good asymptotic behavior, though RHF shows a good performance at the weak correlation limit. (Results corresponding to some other NOFAs like the Goedecker and Umrigar [7], Marques and Lathiotakis [12], and Gritsenko, Pernal and Baerends [10] have not been included due to showing catastrophic performance even for the 2 sites case.) MBB and Power approximations are known to violate the $N$-representability P-, Q-, and G-conditions [20]; as some of us have recently shown [42], even for the two-electron case. $E / t$ values corresponding to the Power functional differ from the ones reported by Kamil et al. (see Fig. 1 in reference [26]). The difference between Kamil's et al. energies and ours is due to the fact that we stick to a spin restricted formalism for our optimizations of the orbitals, so the $\sigma_{g}$ and $\sigma_{u}$ basis is not altered. Therefore, the orbitals for $\alpha$ and $\beta$ electrons were the same. Of course, the usage of an unrestricted formalism improves the $E / t$ values, as Kamil et al. shown, but the price that we have to pay using an unrestricted formalism is that $\left\langle S^{2}\right\rangle \neq 0$ since a mixture between the singlet and triplet solutions may arise (also known as the lost of the exact nonmagnetic character). In order to conserve $\left\langle S^{2}\right\rangle=0$, we have to use a restricted formalism and expect that the accuracy of the NOFA produces the correct asymptotic behavior in the $U / t \rightarrow \infty$ limit (which is not the case of the Power functional as illustrated in the left-top of Fig. 4).

The robustness of well-behaved functionals has been tested beyond the dimer using a system with more degrees of freedom such as the half-filled 4 sites square Hubbard model. A similar problem, the $H_{4}$ molecule, has been recently studied in a NOFT context [55]. Contrary to what happened for the 2 sites homogeneous Hubbard model (where intra-pair nondynamic correlation effects were dominant), there is correlation between pairs in the 4 sites square Hubbard model. Therefore, a good functional for the 4 sites square homogeneous Hubbard model should have a good description of nondynamic intra-pair correlation effects but also a reasonable description of inter-pair correlation. Similar to the results obtained for the 2 sites Hubbard model, $E^{N O F A} / t-E^{F C I} / t$ values displayed in the left-middle of Fig. 4 obtained with RHF and Power present bad asymptotic curves, so they do not correspond to a solution of the problem. Conversely, all other NOFAs show proper behavior for small and large $U / t$ values. PNOF7 is in outstanding agreement with FCI for all correlation regimes, whereas CGA differs from the exact curve in the interval $0<U / t<10$. PNOF5, $\mathrm{MBB}$, and CA yield qualitatively correct curves, but their corresponding energies are not as accurate as the ones obtained with CGA and PNOF7. It must be emphasized the importance of inter-pair correlation in order to describe properly this system, because the only difference between PNOF5 and PNOF7 is exclusively due to the addition of a term to account for inter-pair electron correlation.

The half-filled 6 sites hexagone Hubbard model presents an added complexity with respect to the 2 and 4 sites systems. This model has been previously used as a benchmarking, e.g. for testing new approaches based on coupled cluster methods like CCD0 in order to retrieve nondynamic correlation effects [23]. PNOF7 provides the most accurate total energies as illustrated in the left-bottom of Fig. 4, followed closely by CGA. Both 

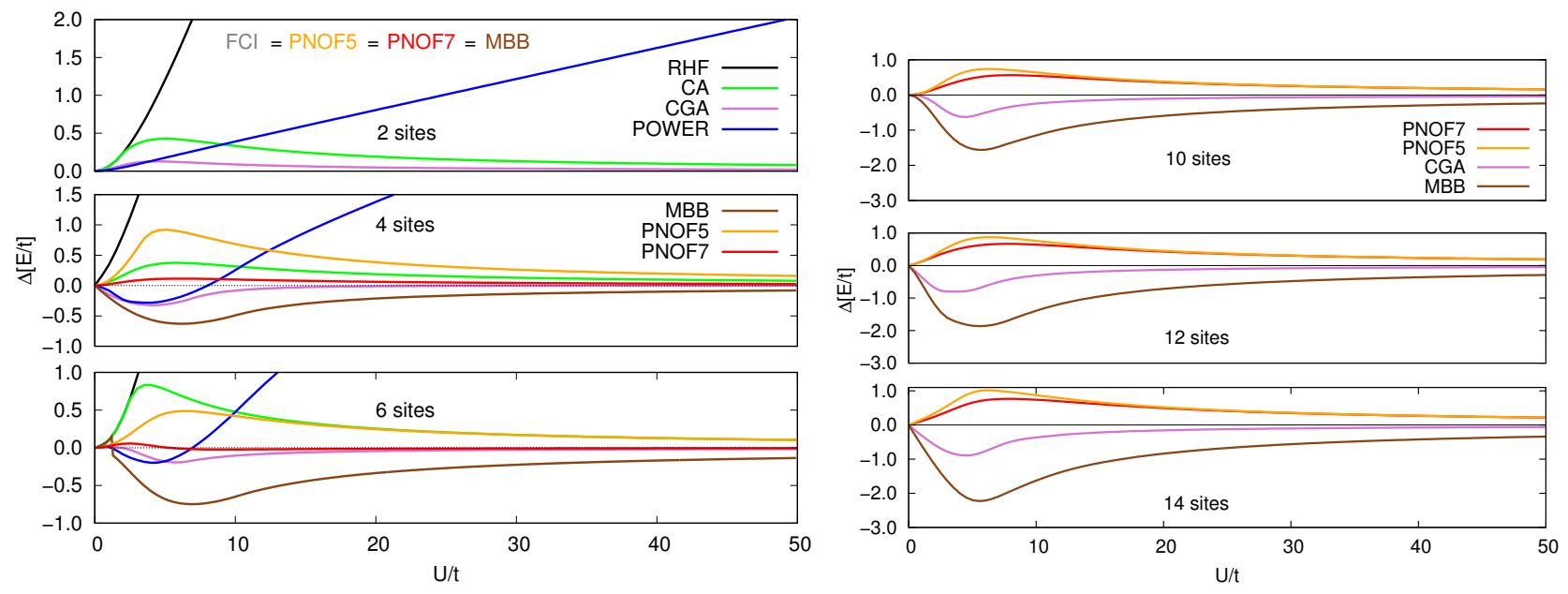

Figure 4: Differences in $E / t$ values with respect to exact FCI obtained for the 1D homogeneous Hubbard model by using many NOFAs.

functionals yield energies slightly below the FCI energy beyond a certain $U / t$ value, so even PNOF7, which is built imposing the analytic necessary $N$-representability conditions of the 2RDM [20,41], is not stricly $N$ representable in constrast to PNOF5. CA, MBB, and PNOF5 show a correct behavior in the weak and strong correlation limits, however they yield poor results in the intermediate region.

Within the limitations imposed by the exact diagonalization calculation, results for larger systems have been obtained, namely, the 10,12, and 14 sites homogeneous Hubbard. Thus, results obtained for PNOF7, PNOF5, $\mathrm{CGA}$, and $\mathrm{MBB}$ approximations are shown in the right hand plots of Fig. 4, whereas other NOFAs have been omitted due to present bad performance for tiny systems. Contrary to the errors shown for smaller systems, CGA yields larger errors going from 10 to 14 sites, as $\mathrm{MBB}$, and both of them show energies below the exact one throughout all energy curve so the variational principle is strongly violated. PNOF approximations do not breakdown for large systems, and in spite of providing an error larger than CGA in absolute terms near the $U / t \approx 50$, they approach closely from above to the exact result for any correlation regime.

Let us focus now on the results obtained for the double occupancy in the case of the 2, 4, and 6 sites models. Since PNOF7 and CGA yield best energies, $d E^{N O F A} / d U-d E^{F C I} / d U$ differences obtained employing these approximations are plotted in Fig. III B. Results obtained by using RHF and PNOF5 are also included for comparison. Note that RHF gives a constant value for each model independently of the site interaction strength $U$. PNOF7 is the only functional able to go parallel to the exact $d E / d U$ according to Fig. IIIB, whereas CGA shows large errors and discontinuities for the 2 and 4 sites systems at small $U / t$ values, which is the region where it goes noticeably below the exact ener-

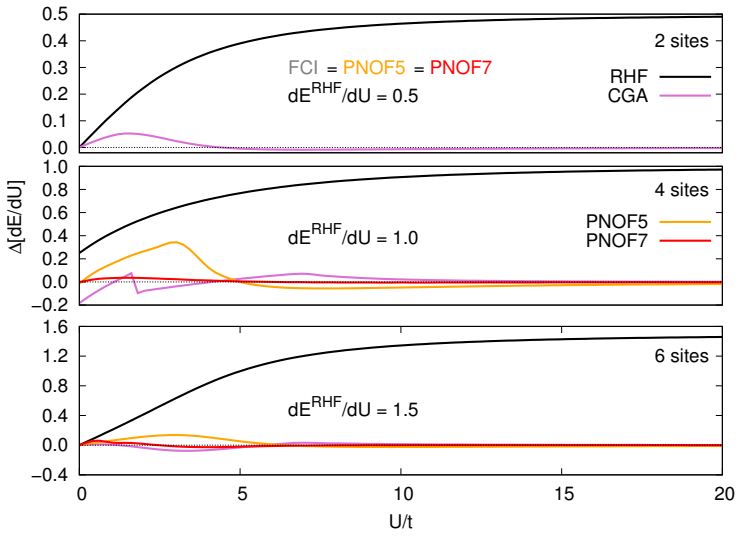

Figure 5: Differences in $d E / d U$ values with respect to exact FCI obtained for many NOFAs.

gies and, as it is shown below, CGA gives wrong pinned natural ONs. The independent pair model PNOF5 fails significantly for systems beyond two particles, specially in the 4-sites case due to the missing inter-pair correlation. This functional shows a behavior similar to RHF for small $U$ values, what can be associated with the result obtained for the natural ONs (see below).

NO occupancies obtained for FCI, CGA, PNOF5, and PNOF7 in the 4 sites square and 6 sites hexagone Hubbard systems are plotted in Fig. 6. Results corresponding to the Hubbard dimer have been omitted since MBB, CGA, PNOF5 and PNOF7 reproduce the exact occupancies. Regarding the result obtained for the 4 sites model, only PNOF7 is able to provide precise ONs for any interaction strength, in contrast to the rest of NOFAs, which show good asymptotic behavior in the $U / t \rightarrow \infty$ limit but tend to stick to $f_{a_{1 g}}=2.0$ (spins summed) occupancies in the weak correlation region related to the bad 


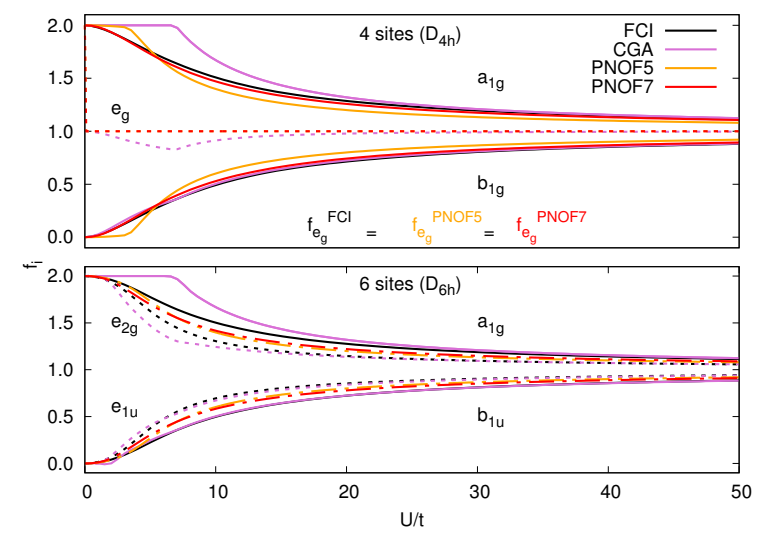

Figure 6: Natural orbital occupancies (spins summed) obtained for FCI, CGA, PNOF5, and PNOF7, in the 4-sites square and 6-sites hexagone Hubbard systems. Solid lines are used for non-degenerate occupancies, whereas dotted and dashed-dotted lines correspond to degenerate occupancies.

performance obtained for $d E / d U$ in the same correlation region. CGA exhibit the largest occupancies pinned to 2.0 in the $a_{1 g}$ orbital but the changes of $b_{1 g}$ occupancy, so it shows a wrong break of the unitary occupation of the two-fold degenerate $e_{g}$ orbitals that remain fixed in the exact solution. Pinned natural ONs are intimately related with the wrong description of the double occupancy. According to Eq. (11), a bad description of the latter is due to an incorrect $2 \mathrm{RDM}$ needed to evaluate $\left\langle\Psi\left|n_{\mu, \uparrow} n_{\mu, \downarrow}\right| \Psi\right\rangle$ in the sites basis, so the double occupancy of the sites computed from first derivative of the energy also reflects the pitfalls in the 2RDM built from pinned to 2.0 ONs.

The situation is completely different for the 6 sites model, for which NOs corresponding to the ground state for both PNOF5 and PNOF7 depend on each approximation, whereas the NOs are still those adapted to the symmetry for the $\mathcal{J K}$-only NOFAs. Thus, PNOF NOs present 3-fold degeneracy and thereby are not related to $D_{6 h}$ point group symmetry, conversely, there is a double degeneracy of the occupations associated with the $e_{2 g}$ and $e_{1 u}$ orbitals for the rest of approximations. Similar to the results obtained for the 4 sites Hubbard model, CGA exhibits pinned occupancies at the weak correlation regime and thereby is not able to give an accurate description of the model. All of the NOFAs shown tend to unitary ONs in the strong correlation limit.

The 3-fold degeneracy can be explained considering that PNOFi $\{i=5,7\}$ functionals lead generally to localization of the molecular orbitals in the NO representation. Nevertheless, there is an equivalent canonical representation that can afford delocalized molecular orbitals adapted to the symmetry of the system upon diagonalization of the matrix of Lagrange multipliers ( $\lambda$ in Eq. 8) obtained from optimized NOs [56]. Thus, the NOs obtained by using the $\mathcal{J K} \mathcal{L}$-only approximations, which are plotted on the right side of Fig. 7, transform into the

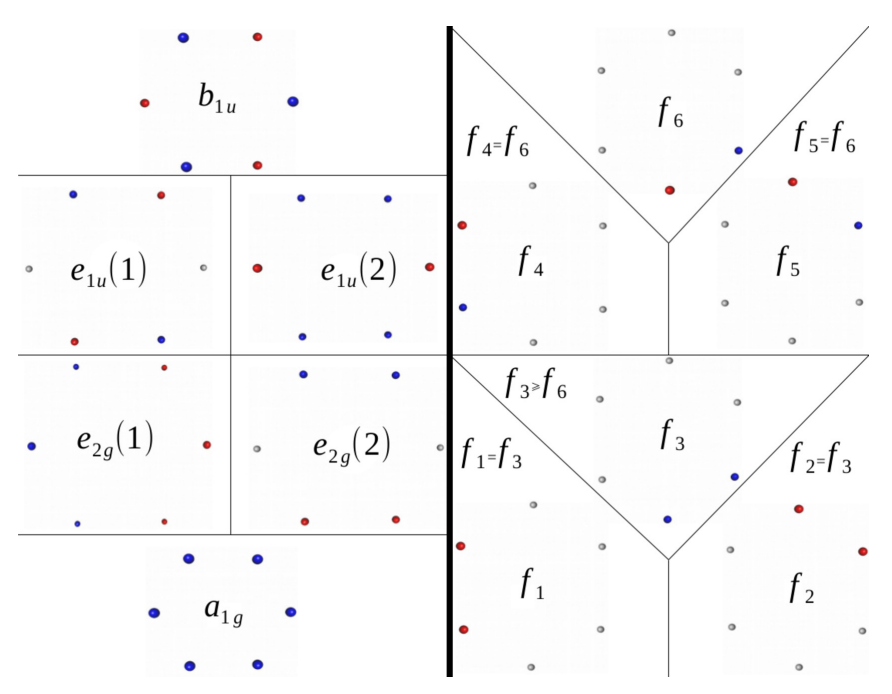

Figure 7: Orbitals obtained by PNOF5 and PNOF7 for the 6 -sites hexagone Hubbard model. Natural orbitals are shown on the right side, while symmetry adapted orbitals (obtained from diagonalization of matrix $\lambda$ ) are plotted in the left side.

symmetry-adapted orbitals shown on the left side of Fig. 7 , so PNOFi $\{i=5,7\}$ functionals are able to retrieve the orbitals adapted to the symmetry of the system in the canonical orbital representation [56]. The 3-fold degeneracy is only a matter of the nature of PNOFi $\{i=5,7\}$ functionals but does not introduce any artifacts in the description of properties.

\section{Inhomogeneous Hubbard model}

MBB, PNOF5 and PNOF7 reproduce the exact results for the energy, double occupancy of sites, and NO occupancies in the homogeneous 2 sites Hubbard model. Nevertheless, the MBB functional does not really recover the exact expression of Löwdin-Shull [39, 57-59] for any 2-electron system (appart from some phases [58]), but PNOFi ( $i=5$ and 7 ) functionals do. In order to prove the deviation of MBB functional and that PNOFi $(i=5$ and 7$)$ actually recover the exact 2-electron functional, we include two additional tests for these approximations. Results obtained by using the CGA approximation are also included to test whether the accuracy of the method still holds. The tests proposed use a inhomogeneous 2 sites Hubbard model, so a non-zero on-site energy is set such that it gives rise to a potential difference $\Delta v=v_{S_{B}}-v_{S_{A}}=2 v$. Since the system is nevermore symmetric the orbitals adapted to the symmetry are no longer the NOs, so the latter may arise from optimization of the energy functional $E\left[\left\{f_{i}\right\}, \phi_{i}(\boldsymbol{x})\right]$ for each NOFA. This model is being used also as benchmarking [24], e.g. for the widely use Bethe ansatz local density approximation (BALDA) developed by Capelle, K. and collaborators [60-64] (which fails recovering the exact results). Then, we have compared the exact results with 

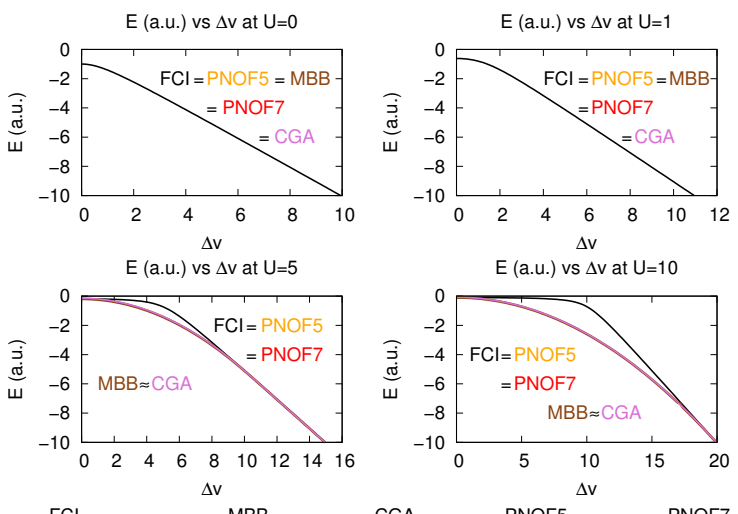

Figure 8: Exact and approximate (obtained with MBB, CGA and PNOF approximations) energies for the inhomogeneous 2 sites Hubbard model for fixed values of $U(U=0,1,5$ and 10) and fixed $t=0.5$.
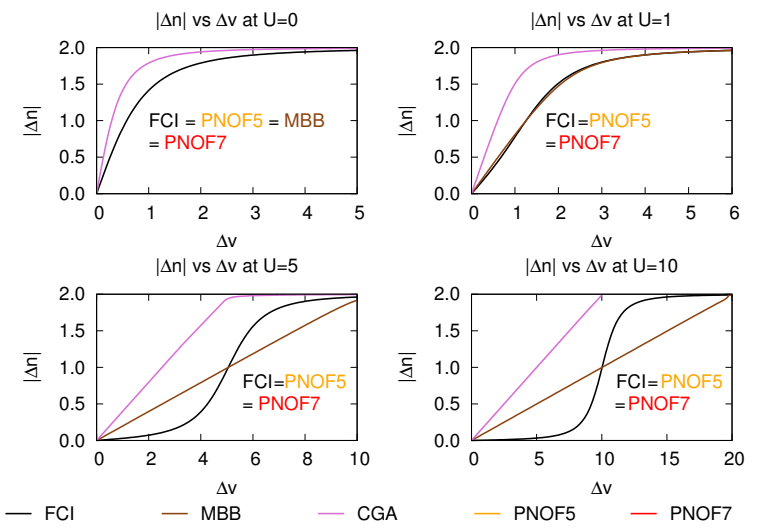

Figure 9: Exact $|\Delta n|$ vs $\Delta v$ and approximated values (obtained with MBB, CGA and PNOF approximations) for the inhomogeneous 2 sites Hubbard model for fixed values of $U$ $(U=0,1,5$ and 10) and fixed $t=0.5$.

the results obtained by using these NOFAs for the total energy and for the difference between site occupancies (i.e. $|\Delta n|=\left|n_{S_{B}}-n_{S_{A}}\right|$ where $n_{S_{X}}$ is the occupancy of site $X)$.

In Fig. 8 we have plotted for a fixed value of $t=0.5$ and four values of $U$ the exact energies and the energies obtained with MBB, CGA, PNOF5 and PNOF7. In general, the $\Delta v=0$ limit recovers the homogeneous 2 sites Hubbard model where all functionals produce exact energies for any $U$ as we observe in Fig. 8. For $U=0$ the exact energy is given by $E=-\sqrt{(2 t)^{2}+(\Delta v)^{2}}$ where the RHF energy is exact since there are no electron-electron interactions for any $\Delta v$, hence MBB, CGA, PNOF5 and PNOF7 reproduce the exact Hartree-Fock energy. Nevertheless, once the on-site interaction is turned on, MBB and CGA do not longer produce exact results for the 2 sites inhomogeneous Hubbard model and only PNOF5 and PNOF7 yield exact energies.

Despite the energy obtained with MBB and CGA func-

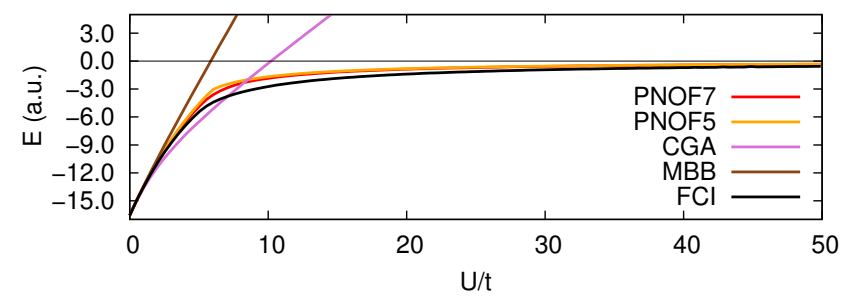

Figure 10: Exact and approximate energies for the 10 sites Hubbard model including an Aubry-André on-site potential, for many $U / t$ values.

tionals for $U=1$ (top right plot in Fig. 8) seems to be still exact, a property such as the $|\Delta n|$ reveals that MBB and CGA results are no longer exact (see Fig. 9). In Fig. 9 we have plotted $|\Delta n|$ for $t=0.5$ and the same four values of $U$ that we used in Fig 8. We observe from the $|\Delta n|$ test that MBB and CGA only yield exact results in the non-interaction limit $(U=0)$, even in the weak correlation region $(U=1) \mathrm{MBB}$ and CGA $|\Delta n|$ values differ from the exact ones. A remarkable result is that PNOF5 and PNOF7 have proven to retrieve the exact functional of Löwdin-Shull. Thus, energies and properties, like for example $|\Delta n|$ vs $\Delta v$ or the charge-transfer to Mott-insulator transitions (which happens around $\Delta v \approx U$ ), obtained with PNOF5 and PNOF7 in any correlation regime for the 2 sites inhomogeneous Hubbard model will be exact.

The on-site potential can be more interestingly modulated if a system with more sites is employed. Hence, we have used an oscillatory potential such as the introduced in the Aubry-André model [49] to carry out calculations on the 10 sites inhomogeneous Hubbard model. Note that the Hamiltonian of the system is now given by Eq. (10) with $\alpha=1 / 10, V=2.0$ and $\delta=-2 \pi / 10$. According to the results given in Fig. III C, and similar to the results obtained for the inhomogeneous Hubbard dimer, CGA and MBB approximations fail dramatically when studying inhomogeneous systems except in the weak correlation limit. PNOF5 and PNOF7 approximations not only show good asymptotic behavior, but they lie close to the exact curve for any correlation regime. Therefore, systems with spatial inhomogeneities are also welldescribed by PNOF approximations.

\section{CONCLUSION}

The resurgence of interest in reduced density-matrix functionals has brought a new class of theoretical methods that appeared recently in the literature [5-18]. These approximations are given in terms of natural orbitals (NOs) and occupation numbers (ONs), so they should be referred to as Natural Orbital Functional approximations (NOFAs). It must be distinguish between NOFAs 
developed with simple assumptions about the hole function, e.g. MBB, Power, CA, or CGA approximations; and NOFAs that are built imposing necessary known $N$ representability conditions of the $2 \mathrm{RDM}$, such as PNOF5 or PNOF7. In this paper the robustness of a set of NOFAs is put into test by using the homogeneous Hubbard model with 2, 4, 6, 10, 12, and 14 sites, and the inhomogeneous model which includes nonzero on-site potential for the Hubbard model with 2 and 10 sites. A simple comparison between the exact FCI calculations and approximated results for varying interaction strengths $U / t$ is carried out.

Both PNOF5 and PNOF7 reproduce the exact LöwdinShull functional for the 2 sites system, while the other NOFAs fail for one or another test. Consistently for all test cases studied in the homogeneous Hubbard model PNOF7 results are in outstanding agreement with FCI. Among $\mathcal{J K}$-only NOFAs only CGA is able to provide energies close to the exact ones, but fails significantly for ONs of the NOs, and yield a discontinuous curve for the double occupancy of the sites. The enhanced accuracy of CGA is in fact due to the inclusion of a proper particle-hole symmetry which is not the case for some other $\mathcal{J K}$-only NOFAs. Nevertheless, CGA violates many fundamental properties such as $N$-representability conditions or antisymmetry of the 2RDM [42]. Thus, CGA fails dramatically for the inhomogeneous Hubbard model, i.e when the on-site potential varies. PNOF7 presents particle-hole symmetry at the same time that the fundamental properties of the 2RDM are conserved, and thereby the inhomogeneous Hubbard is also well described.

In view of the results obtained for the Hubbard model with varying interaction strength, PNOF7 can describe not only weakly correlated systems, but also problems where strong correlation effects arise. This opens a new avenue where PNOF7 becomes a tool for studying many interesting applications which include con- fined fermions, disorder and critical behavior in optical lattices, effects of spatial inhomogeneity in strongly correlated systems, various critical phenomena in 1-D chains, among others. Where up to now, appart from the well-established variational wavefunction methods of Gutzwiller and Baeriswyl, different perturbative expansions, or Quantum Monte-Carlo methods, the widely used BALDA approximation has lead, despite the pitfalls obtained with it even in the simple 2 sites nonsymmetric Hubbard model [24].

To conclude, the present study proves the importance of developing functionals that satisfy at least with the analytic necessary $N$-representability conditions of the 2RDM in order to obtain consistent results in systems with either weak or strong electronic correlation. Besides, concerning the improvement of PNOF7 over the independent pair model PNOF5, it shows that a well-balanced inter-pair correlation is crucial to account properly for electron correlation effects. Our calculations shines light on the insight, successes, and limits of current NOFT approaches, and may be useful for the development of new natural orbital functional approximations.

\section{Acknowledgements}

Financial support comes from Eusko Jaurlaritza (Ref. IT588-13) and Ministerio de Economía y Competitividad (Ref. CTQ2015-67608-P). One of us (I.M.) is grateful to Vice-Rectory for research of the UPV/EHU for the PhD. grant (PIF//15/043). M.R.M. wants to thank the Spanish Ministry (MEC) for the PhD. grant (FPU-2013/00176), and to the Spanish MINECO/FEDER Projects CTQ2014-52525-P and CTQ2015-67608-P. Also, the authors want to thank Mr. Killian Deur, Mr. Laurent Mazouin, and Mr. Bruno Senjean for the fruitful discussions.
[1] T. L. Gilbert, Phys. Rev. B 12, 2111 (1975).

[2] M. Levy, Proc.Natl. Acad. Sci. USA 76, 6062 (1979).

[3] R. A. Donnelly, J. Chem. Phys. 71, 2874 (1979).

[4] S. M. Valone, J. Chem. Phys. 73, 1344 (1980).

[5] A. M. K. Müller, Phys. Lett. 105A, 446 (1984).

[6] M. A. Buijse, Ph.D. thesis, Vrije Universiteit, Amsterdam, The Netherlands (1991).

[7] S. Goedecker and C. J. Umrigar, Phys. Rev. Lett. 81, 866 (1998).

[8] G. Csányi and T. A. Arias, Phys. Rev. B 61, 7348 (2000).

[9] G. Csányi, S. Goedecker, and T. A. Arias, Phys. Rev. A 65, 032510 (2002).

[10] O. Gritsenko, K. Pernal, and E. J. Baerends, J. Chem. Phys. 122, 204102 (2005).

[11] M. Piris, Int. J. Quantum Chem. 106, 1093 (2006).

[12] M. A. L. Marques and N. N. Lathiotakis, Phys. Rev. A 77, 032509 (2008).

[13] S. Sharma, J. K. Dewhurst, N. N. Lathiotakis, and
E. K. U. Gross, Phys. Rev. B 78, 201103(R) (2008).

[14] M. Piris, X. Lopez, F. Ruipérez, J. M. Matxain, and J. M. Ugalde, J. Chem. Phys. 134, 164102 (2011).

[15] M. Piris, J. M. Matxain, and X. Lopez, J. Chem. Phys. 139, 234109 (2013).

[16] M. Piris, J. Chem. Phys. 141, 044107 (2014).

[17] M. Piris, Phys. Rev. Lett. (in press).

[18] M. A. Buijse and E. J. Baerends, Mol. Phys. 100, 401 (2002).

[19] E. J. Baerends, Phys. Rev. Lett. 87, 133004 (2001).

[20] D. A. Mazziotti, Phys. Rev. Lett. 108, 263002 (2012).

[21] M. Piris, J. Math. Chem. 25, 47 (1999).

[22] M. F. Silva, N. A. Lima, A. L. Malvezzi, and K. Capelle, Phys. Rev. B - Condensed Matter and Materials Physics 71, 1 (2005).

[23] I. W. Bulik, T. M. Henderson, and G. E. Scuseria, J. Chem. Theory Comput. (2015).

[24] D. J. Carrascal, J. Ferrer, J. C. Smith, and K. Burke, 
J. Phys.: Condens. Matter 27, 393001 ((2015) and references therein).

[25] S. Di Sabatino, J. A. Berger, L. Reining, P. Romaniello, and S. D. Sabatino, J. Chem. Phys. 143, 24108 (2015).

[26] E. Kamil, R. Schade, T. Pruschke, and P. E. Blöchl, Phys, Rev. B 93, 085141 (2016).

[27] M. C. Gutzwiller, Phys. Rev. Lett. 10, 159 (1963).

[28] M. C. Gutzwiller, Phys. Rev. 137, A1726 (1965).

[29] D. Baeriswyl, Found. Phys. 30, 2033 (2000).

[30] M. Dzierzawa, D. Baeriswyl, and M. Martelo, Helv. Phys. Acta 70, 124 (1997).

[31] D. Baeriswyl, in Nonlinearity in Condensed Matter, edited by A. R. Bishop, D. K. Campbell, P. Kumar, and S. E. Trullinger (Springer-Verlag, 1986).

[32] J. R. Hammond and D. A. Mazziotti, Phys. Rev. A 73, 062505 (2006).

[33] N. C. Rubin and D. A. Mazziotti, Theor. Chem. Acc. 133, 1492 (2014).

[34] Y. Shinohara, S. Sharma, J. K. Dewhurst, S. Shallcross, N. N. Lathiotakis, and E. K. U. Gross, New J. Phys. 17, 093038 (2015).

[35] K. Pernal, New J. Phys. 17, 111001 (2015).

[36] E. V. Ludeña, F. J. Torres, and C. Costa, J. Mod. Phys. 04, 391 (2013).

[37] M. Piris, in Reduced-Density-Matrix Mechanics: with applications to many-electron atoms and molecules, edited by D. A. Mazziotti (John Wiley and Sons, Hoboken, New Jersey, USA, 2007), chap. 14, pp. 387-427.

[38] M. Piris and J. M. Ugalde, Int. J. Quantum Chem. 114, 1169 (2014).

[39] K. Pernal and K. J. H. Giesbertz, Top. Curr. Chem. 368, 125 (2016).

[40] A. J. Coleman, Rev. Mod. Phys. 35, 668 (1963).

[41] J. M. Herbert and J. E. Harriman, J. Chem. Phys. 118, 10835 (2003).

[42] M. Rodríguez-Mayorga, E. Ramos-Cordoba, M. ViaNadal, M. Piris, and E. Matito (submitted).

[43] D. A. Mazziotti, Chem. Phys. Lett. 289, 419 (1998).

[44] M. Piris, J. M. Matxain, X. Lopez, and J. M. Ugalde, J. Chem. Phys. 131, 021102 (2009).
[45] M. Piris, Int. J. Quantum Chem. 113, 620 (2013).

[46] M. Piris and J. M. Ugalde, J. Comput. Chem. 30, 2078 (2009).

[47] D. A. Mazziotti, J. Chem. Phys. 112, 10125 (2000).

[48] D. A. Mazziotti, Chem. Phys. Lett. 338, 323 (2001).

[49] H. Z. Shen, X. X. Yi, and C. H. Oh, J. Phys. B: Atomic, Molecular and Optical Physics 47, 085501 (2014).

[50] P. J. Knowles and N. C. Handy, Chem. Phys. Lett. 111, 315 (1984).

[51] P. J. Knowles and N. C. Handy, Comput. Phys. Commun. 54, 75 (1989).

[52] E. Matito and F. Feixas, DMn program (2009), university of Girona (Spain) and University of Szczecin (Poland).

[53] A. Akande and S. Sanvito, Phys. Rev. B 82, 245114 (2010).

[54] Y. Shinohara, S. Sharma, S. Shallcross, N. N. Lathiotakis, and E. K. U. Gross, J. Chem. Theory Comput. 11, 4895 (2015).

[55] E. Ramos-Cordoba, X. Lopez, M. Piris, and E. Matito, J. Chem. Phys. 143 (2015).

[56] M. Piris, J. M. Matxain, X. Lopez, and J. M. Ugalde, Theor. Chem. Acc. 132, 1298 (2013).

[57] P.-O. Löwdin and H. Shull, Phys. Rev. Lett. 101, 1730 (1956).

[58] K. Pernal and J. Cioslowski, J. Chem. Phys. 120, 5987 (2004).

[59] J. Cioslowski and K. Pernal, Phys. Rev. Lett. 430, 188 (2006).

[60] N. A. Lima, L. N. Oliveira, and K. Capelle, EPL (Europhysics Letters) 60, 601 (2002).

[61] N. A. Lima, M. F. Silva, L. N. Oliveira, and K. Capelle, Phys. Rev. Lett. 90, 146402 (2003).

[62] K. Capelle, N. Lima, M. Silva, and L. Oliveira, vol. 14 (Springer Netherlands, 2003).

[63] G. Xianlong, M. Polini, M. P. Tosi, V. L. Campo, and K. Capelle, Phys. Rev. B 73, 165120 (2006).

[64] V. V. França, D. Vieira, and K. Capelle, New J. Phys. 14, 073021 (2012). 